\title{
RELATIONSHIP BETWEEN MICROEMULSION STRUCTURE AND SURFACTANT LAYER BENDING ELASTICITY
}

\author{
L.T. LEE ${ }^{\mathrm{a}}$, D. LANGEVIN ${ }^{\mathrm{a}, 1}$ and R. STREY ${ }^{\mathrm{b}}$ \\ ${ }^{2}$ Laboratoire de Physique Statistique, Ecole Normale Supérieure, 24 rue Lhomond, \\ 75231 Paris Cedex 05, France \\ ${ }^{\mathrm{b}}$ Max Planck Insitut für Biophysikalische Chemie, D-3400 Göttingen, Fed. Rep. Germany
}

\begin{abstract}
We recall briefly the knowledge on microemulsion structure emphasizing the importance of the surfactant layer properties. We present results of experiments made with water-alkanenon-ionic surfactants systems that, together with results obtained earlier in other systems, confirm the close relationship between the maximum characteristic size in the microemulsion and the persistence length of the surfactant layer. The possible influence of the Gaussian bending elastic modulus is discussed.
\end{abstract}

\section{Introduction}

Microemulsions are thermodynamically stable mixtures of oil and water, stabilized by surfactant molecules $[1,2]$. Their structure is frequently made of droplets, either of oil or water, surrounded by a surfactant monolayer. The nature of the external phase depends on the spontaneous curvature $C_{0}$ of the surfactant monolayer: if $C_{0}$ is positive (resp. negative), the layer tends to curve towards water (resp. oil) and an oil in water (o/w) (resp. a water in oil, (w/o)) microemulsion is obtained if the amount of oil (resp. water) is small. The droplet radius is given by

$$
R=3 \phi / C_{\mathrm{s}} \Sigma
$$

where $\phi$ is the dispersed phase volume fraction, $C_{\mathrm{s}}$ the surfactant concentration (number of molecules per unit volume) and $\Sigma$ the area per surfactant molecule in the monolayer. It has been experimentally found that $\Sigma$ is relatively constant for a given surfactant: this supports the assumption that the surfactant layer is compact and rather incompressible [1].

When the oil (resp. water) amount is increased, the droplet radius increases according to eq. (1), and reaches the spontaneous radius of curvature $R_{0}=$

\footnotetext{
${ }^{1}$ Author to whom correspondence should be addressed.
} 
$\left|C_{0}^{-1}\right|$. At this point, when more oil is added, this oil is rejected in an excess phase. This is called the emulsification failure [3]. In some cases, phase separation occurs ealier: when the droplet radius increases, attractive interactions between the droplets can become important, and a phase separation between two microemulsion phases can be observed. The behaviour is analog to critical phenomena in simpler fluids.

When the spontaneous curvature $C_{0}$ is small, a different type of structure is obtained. In the absence of thermal fluctuations, the structure would be lamellar: oil and water layers separated by surfactant monolayers. These structures are indeed observed in oil-water-surfactant mixtures, but they are distinct from microemulsion phases: due to their different symmetry, they are separated from these phases by first order transitions. In other cases, microemulsion phases are also obtained for small $\left|C_{0}\right|$ values: as in droplet microemulsions they are isotropic phases. Unlike lamellar phases, they are continuously connected to droplet microemulsion phases. But they are random, sponge-like oil and water continuous, i.e. bicontinuous phases. From random space-filling models, the mean size $\xi$ of the oil and water microdomains can be calculated. For a cubic lattice

$$
\xi=6 \phi_{\mathrm{o}} \phi_{\mathrm{w}} / C_{\mathrm{s}} \Sigma
$$

where $\phi_{\mathrm{o}}$ and $\phi_{\mathrm{w}}$ are respectively the oil and water volume fractions. $\xi$ increases with $\phi_{\mathrm{o}}$ and $\phi_{\mathrm{w}}$ and reaches a maximum $\xi_{0}$, above which the oil and water are rejected as excess phases: a generalization of the emulsification failure concept.

The fact that for a given set of conditions (temperature, chemical potential, composition) the system displays well defined structures (w/o, o/w and bicontinuous) suggests that one can attribute a bending energy to the interfacial layer of the system. This energy per unit area can be written as [4]

$$
F=\frac{1}{2} K\left(C_{1}+C_{2}-2 C_{0}\right)^{2}+\bar{K} C_{1} C_{2},
$$

where $C_{1}$ and $C_{2}$ are the local principal curvatures of the surfactant layer and $K$ and $\bar{K}$ are respectively the splay and the Gaussian bending elastic moduli. The contribution of $F$ to the total free energy is fundamental in the determination of the type and of the characteristic size of the microemulsion structure. In the following, $\bar{K}$ will be assumed to be zero unless stated otherwise.

When $C_{0}$ is large in absolute value, we have seen that the maximum droplet size is close to $1 / C_{0}$. When $C_{0} \sim 0$ and $K$ is of order $k T$, this is no longer the case. The monolayers are locally flat but strongly undulated due to thermal motion. The cutoff wavelength of the thermal fluctuations is the persistence 
length [1]:

$$
\xi_{K}=a \exp (2 \pi K / k T)
$$

$a$ being a molecular length. $\xi_{K}$ is the correlation length for the normals to the layer, i.e. the distance over which the layer remains flat.

Experiments show that bicontinuous microemulsions are not completely random: there is a rather well defined characteristic distance between the oil and water microstructural elements. This distance corresponds to the mean size in the space filling models, as given by eq. (2). There are now two types of theories attempting to give a physical significance to $\xi_{0}$, the maximum value of $\xi$. Indeed, because of the strong thermal fluctuations, $\xi_{0}$ is more likely to be related to $\xi_{K}$ than to $C_{0}^{-1}$.

In the first type of theories, the free energy of the system is calculated by incorporating the dispersion entropy, the interfacial energy and the bending energy (eq. (3)). Since bicontinuous microemulsions can coexist with both excess oil and water, these theories have to account for three phase equilibria. This can be done by assuming that the surfactant film is not fully incompressible (the interfacial energy accounts for the $\Sigma$ variations) $[5,6]$. The relationship between $\xi_{0}$ and $\xi_{K}$ obtained in this way is rather indirect. A second approach can be made by assuming that the surfactant monolayer is incompressible but that the elastic constant varies with the scale, as recently demonstrated [7,8]. In this way, $\xi_{0}$ is found to be proportional to $\xi_{K}[9]$.

The second type of theories are lattice theories, in which a rigourous treatment of the thermal fluctuations can be made more easily [10-12]. However a phenomenological microscopic bending elasticity has to be used and the connections between the results and the macroscopic notion of persistence length is still far from clear.

In this paper, we will present experimental determination of microemulsion microstructures and of the surfactant film macroscopic bending elasticity, which strongly support the close relationship between $\xi_{0}$ and $\xi_{K}$.

\section{Experimental}

The microemulsions studied here are mixtures of water, alkanes and nonionic surfactants, alkyl polyglycol ethers $\left(\mathrm{C}_{i} \mathrm{E}_{j}\right)$. Three surfactants of increasing chain lengths have been chosen in order to vary the bending elastic constant of the surfactant layer: $\mathrm{C}_{8} \mathrm{E}_{3}$ (from Bachem), $\mathrm{C}_{10} \mathrm{E}_{4}$ and $\mathrm{C}_{12} \mathrm{E}_{5}$ (from Nikko Chemicals). The corresponding alkanes are decane, octane and hexane, respectively. In this way, the temperatures at which middle phase microemulsions are 
obtained fall in the same range. For $T<T_{1}$ one obtains an $\mathrm{o} / \mathrm{w}$ microemulsion coexisting with excess oil, for $T>T_{\mathrm{u}}$ a w/o microemulsion coexisting with excess water, and for $T_{1}<T<T_{\mathrm{u}}$ a middle phase microemulsion coexisting with both excess oil and water. This is because when the temperature is increased, the hydration water of the ethylene oxide groups is released, thus leading to a decrease in the bulkiness of the surfactant polar part, i.e. to a decrease in $C_{0}$. The composition and transition temperatures for the three mixtures are respectively

\begin{tabular}{|c|c|c|c|c|}
\hline $\mathrm{C}_{8} \mathrm{E}_{3}$ & $4 \mathrm{wt} \%$ & $\begin{array}{l}\text { decane } \\
\text { water }\end{array}$ & $\begin{array}{l}48 \mathrm{wt} \% \\
48 \mathrm{wt} \%\end{array}$ & $\begin{array}{l}T_{1}=16^{\circ} \mathrm{C} \\
T_{\mathrm{u}}=28.5^{\circ} \mathrm{C}\end{array}$ \\
\hline $\mathrm{C}_{10} \mathrm{E}_{4}$ & $2 \mathrm{wt} \%$ & $\begin{array}{l}\text { octane } \\
\text { water }\end{array}$ & $\begin{array}{l}49 \mathrm{wt} \% \\
49 \mathrm{wt} \%\end{array}$ & $\begin{array}{l}T_{1}=22.8^{\circ} \mathrm{C} \\
T_{\mathrm{u}}=28^{\circ} \mathrm{C}\end{array}$ \\
\hline$C_{12} E_{5}$ & $1 \mathrm{wt} \%$ & $\begin{array}{l}\text { hexane } \\
\text { water }\end{array}$ & $\begin{array}{l}49.5 \mathrm{wt} \% \\
49.5 \mathrm{wt} \%\end{array}$ & $\begin{array}{l}T_{1}=26.5^{\circ} \mathrm{C} \\
T_{\mathrm{u}}=28.8^{\circ} \mathrm{C}\end{array}$ \\
\hline
\end{tabular}

They are in agreement with known phase diagrams [13].

Partial phase diagrams for the $\mathrm{C}_{10} \mathrm{E}_{4}$ system in the regions $T<T_{1}$ and $T>T_{\mathrm{u}}$ are shown in fig. 1 [14]. The points represent the single phase limit: emulsification failure. From eq. (1), one can calculate the maximum droplets radius $R_{0}$, i.e. the inverse of the surfactant layer spontaneous curvature. The data are given in table I.

It should be noted that the emulsification failure lines do not extrapolate to the oil corner in the phase diagram triangle: this is because a substantial amount of surfactant is present in the form of monomer in the oil phase. In other words, the critical micellar concentrations $(\mathrm{cmc})$ of these surfactants in oil is large, whereas for water they are quite small.

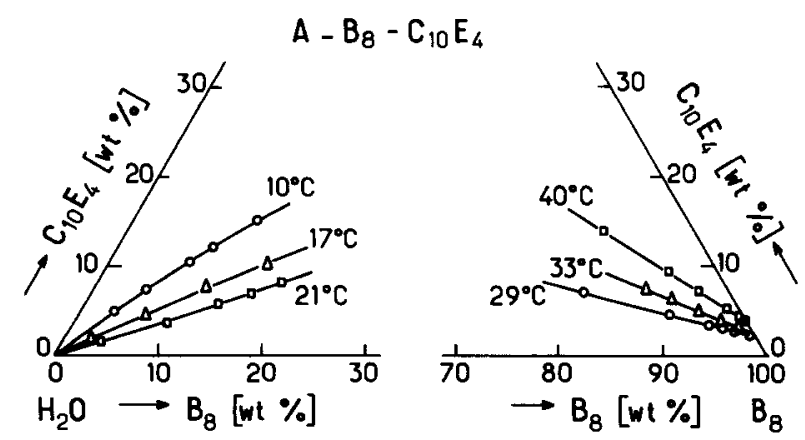

Fig. 1. Phase diagram for the water-octane $\left(B_{8}\right)-C_{10} E_{4}$ at different temperatures. The triangle upper corner is pure surfactant. The scales correspond to wt\%. See also ref. [14]. 
Table I

Calculated droplet radii and oil-water interfacial tensions for the water-octane- $\mathrm{C}_{10} \mathrm{E}_{4}$ system, and measured tensions.

\begin{tabular}{llccc}
\hline$T\left({ }^{\circ} \mathrm{C}\right)$ & Microemulsion & $R_{0}(\AA)$ & $\gamma_{\mathrm{e}}+\gamma_{\mathrm{c}}(\mathrm{mN} / \mathrm{m})$ & $\gamma(\mathrm{mN} / \mathrm{m})$ \\
\hline 10 & o/w & 91 & & \\
17 & & 103 & $5.7 \times 10^{-2}$ & $9 \times 10^{-2}$ \\
21 & & 110 & $4 \times 10^{-2}$ & $3 \times 10^{-2}$ \\
29 & w/o & 97 & $5.2 \times 10^{-2}$ & $2.8 \times 10^{-2}$ \\
33 & & 89 & $6.1 \times 10^{-2}$ & $10^{-1}$ \\
40 & & 81 & & \\
\hline
\end{tabular}

In the region $T_{1}<T<T_{\mathrm{u}}$, the relative volumes of the different phases were measured, and the value of $\xi_{0}$ calculated using eq. (2). The data for $\xi_{0}$ and $R_{0}$ are shown in fig. 2.

Since the spontaneous curvature evolves continuously from positive to negative values, it becomes zero in the inversion temperature range $T_{1}<T<$ $T_{\mathrm{u}}$. The figure clearly shows that $\xi_{0}$ does not become infinite like $C_{0}^{-1}$.

Neutron scattering experiments have been performed on the middle phase microemulsion. In can be shown that because of the correlations between oil and water microdomains, the scattering spectra exhibit a peak at a wave vector $q_{\mathrm{m}}=\pi / \xi[15]$. The peaks are not sharp as in lamellar phases, their width $\Delta q$ are of the order of $q_{\mathrm{m}}$. The $\xi$ values deduced in this way have been plotted in fig. $2[16]$.

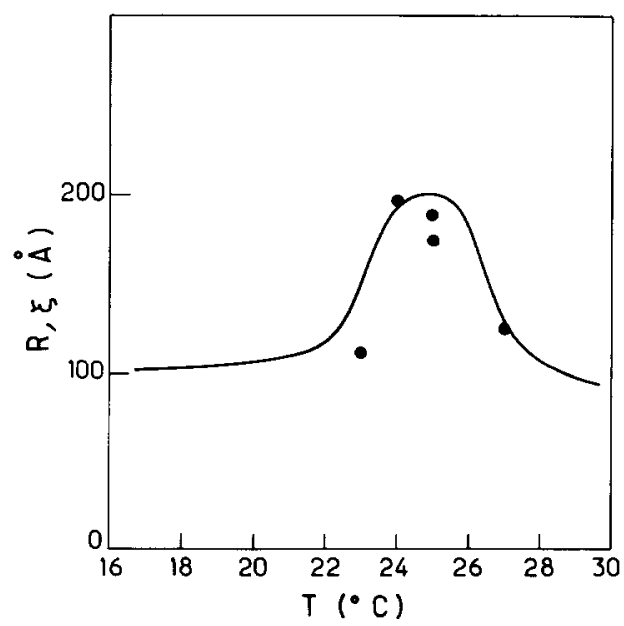

Fig. 2. Calculated droplets radii $R_{0}$ and sizes $\xi_{0}$ (full line) and data from neutron scattering experiments (circles) versus temperature for the $\mathrm{C}_{10} \mathrm{E}_{4}$ system. 
The bending elastic modulus has been measured by ellipsometry. In this technique, the ellipticity $\rho$ of the light reflected at the Brewster angle by an interface between two fluids is determined. For an ideal flat, infinitely thin interface, Fresnel has shown that $\rho=0$. A real interface is thick and rough, and $\rho \neq 0$. We have studied the interfaces between oil and water in our systems. Provided that these phases are saturated in surfactant monomers $\left(C_{\mathrm{s}}>\mathrm{cmc}\right)$, the interface is covered with a surfactant monolayer, the same incompressible monolayer as the one that covers the oil-water interfaces in the microemulsion microstructural elements. The oil-water interfacial tensions $\gamma$ are ultralow in these systems. They have been measured by surface light scattering [16] and are represented in fig. 3. Because of these low tensions, the interfaces are strongly undulated and the ellipticity is dominated by the roughness contribution. The mean square amplitude of the surface fluctuations is

$$
\left\langle\zeta^{2}\right\rangle=\frac{1}{2 \pi} \int \frac{k T q \mathrm{~d} q}{\Delta \rho g+\gamma q^{2}+K q^{4}},
$$

where $\Delta \rho g$ is the gravity contribution. The roughness contribution to the ellipticity is [17]

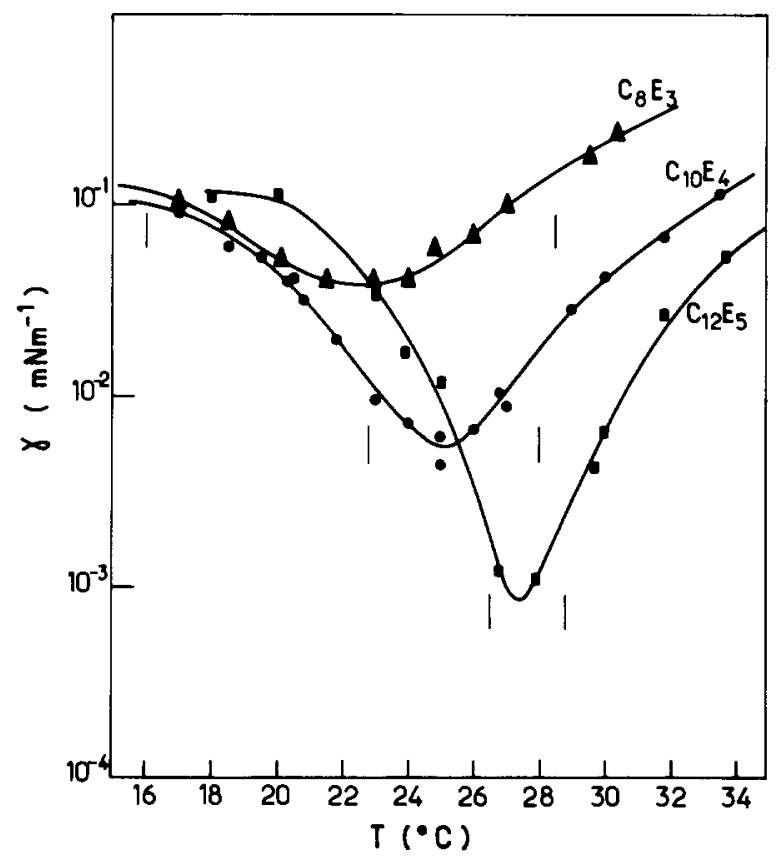

Fig. 3. Interfacial tension versus temperature for the oil water interfaces with non-ionic surfactants monolayers. The vertical bars indicate the limits of the three-phase region. 


$$
\rho=-\frac{3 \pi k T}{8 \lambda} \frac{\varepsilon_{1}-\varepsilon_{2}}{\sqrt{\varepsilon_{1}+\varepsilon_{2}}} \frac{1}{\sqrt{K \gamma}},
$$

where $\varepsilon_{1}$ and $\varepsilon_{2}$ are the dielectric constants of the coexisting phases and $\lambda$ the light wavelength.

The values that we have deduced for $K$ are respectively $0.51 k T$ for $\mathrm{C}_{10} \mathrm{E}_{4}$ and $0.31 k T$ for $\mathrm{C}_{8} \mathrm{E}_{3}[16]$. They are compared with $\mathrm{K}$ values obtained from other systems in table II.

\section{Discussion}

We have mentioned that $K$ is scale dependent. In ellipsometry, we measure $K$ at the cutoff length of capillary waves $l_{c}=(2 / \pi) \sqrt{K / \gamma}$. To calculate the persistence length, the $K$ value at the molecular scale $a$ must be used. When measuring the amplitude of the thermal undulations of a macroscopic interface, we also have to deal with the scale variations of the surface tension, which are correlated with those of $K[16]$. One then obtains a slightly different expression for $\xi_{K}$,

$$
\xi_{K}=2.117 a \exp [3.254 K(a) / k T] \text {. }
$$

$K(l)$ has no simple analytic expression and is given in ref. [17].

In table II, we have reported the values of $K$ and $\xi_{K}$ calculated with $a=7 \AA$, approximately the square root of the area per surfactant molecule. We also give the value of the maximum size $\xi_{0 m}$ measured at the temperature for which $C_{0}=0$ and $\phi_{\mathrm{o}}=\phi_{\mathrm{w}}$. At that temperature, the oil-water interfacial tension is minimum.

The other surfactants in the table are ionic surfactants [18]. The evolution from positive to negative curvature has been obtained by adding variable

Table II

Measured and renormalized bending elastic moduli, maximum size in bicontinuous microemulsions, persistence length of the surfactant layer for different surfactant systems (data from refs. $[16,18])$.

\begin{tabular}{llclcc}
\hline Surfactant & $K\left(l_{\mathrm{c}}\right) / k T$ & $l_{\mathrm{c}}(\AA)$ & $K(a) / k T$ & $\xi_{\text {am }}(\AA)$ & $\xi_{K}(\AA)$ \\
\hline $\mathrm{C}_{8} \mathrm{E}_{3}$ & 0.31 & 36 & 0.35 & 58 & 69 \\
CTAB & 0.4 & 50 & 0.53 & 92 & 83 \\
DTAB & 0.4 & 50 & 0.53 & 95 & 83 \\
$\mathrm{C}_{10} \mathrm{E}_{4}$ & 0.51 & 120 & 0.76 & 187 & 211 \\
SDS & 0.65 & 167 & 0.96 & 230 & 336 \\
SHBS & 0.4 & 200 & 0.82 & 400 & 213 \\
\hline
\end{tabular}


amounts of salt to water, i.e. by progressively screening the electrostatic interactions between the polar parts of the surfactant molecules.

Dodecyltrimethyl ammonium bromide (DTAB) and sodium dodecylsulfate (SDS) have $\mathrm{C}_{12}$ chains, hexadecyltrimethyl ammonium bromide (CTAB) and sodium hexadecyl sulfonate (SHBS) $\mathrm{C}_{16}$ chains. These surfactants form very rigid layers and cannot lead to microemulsion formation unless a short chain alcohol is added. The role of the alcohol is to desorganize the layer to a certain extent, i.e. to decrease it rigidity. This has been confirmed by recent computer simulations [19]. The data of the table correspond to a temperature of $20^{\circ} \mathrm{C}$ and to mixed butanol-surfactant layers for the ionic surfactants [18].

There is a remarkable agreement between the variations of $\xi_{K}$ and the maximum size in the middle phase microemulsions. The only exception is SHBS, which is a double chain surfactant. The area per surfactant molecule is larger than for the other surfactants [20]. One should probably use a large a value for the renormalization calculation, but this will not substantially increase $\xi_{K}$. Let us recall that theory only predicts that $\xi_{0 \mathrm{~m}}$ and $\xi_{K}$ are proportional.

It was shown that although DTAB ( $C_{12}$ chain) has a smaller chain length than CTAB ( $C_{16}$ chain) there is much more alcohol in the CTAB layers (3 molecules of butanol per surfactant molecule) than in the DTAB layer $(1: 1)$ [21]. This explains why the bending elastic modulus remains the same. This also points out the interest of the study of alcohol free systems. Indeed alcohol being soluble in oil and water, its partitioning between the oil, water and surfactant film cannot be controlled.

Let us now comment on the role of the second bending elastic constant $\bar{K}$ associated to Gaussian curvature. When $\bar{K}<0$, the free energy equation (3) indicates that structures where $C_{1}$ and $C_{2}$ have the same sign will be favoured. This is expected to be the case for droplet structures. By minimizing the bending energy at constant total surfactant area, one finds that at the emulsification failure limit

$$
R_{0}=C_{0}^{-1} \frac{2 K+\bar{K}}{2 K}
$$

i.e. $R_{0}$ is smaller than $1 / C_{0}$. When $\bar{K}>0$, a structure containing a number of saddle points ( $C_{1}$ and $C_{2}$ having opposite signs) is favoured. These structures are found in cubic surfactant phases [22].

In our experiments, we measure the amplitude of the thermal fluctuations at a macroscopic interface, where the topology is conserved (no holes in the surfactant layers are expected to be formed). For a given topology, the second term of eq. (3) does not give any contribution. We are therefore measuring $K$ 
with no corrections due to $\bar{K}$. It can be shown theoretically that the interfacial tension between a droplet microemulsion and an excess phase contains a contribution due to the bending elasticity [1]

$$
\gamma_{\mathrm{c}}=\frac{2 K C_{0}}{R_{0}}
$$

which with eq. (8) becomes

$$
\gamma_{\mathrm{c}}=\frac{2 K+\bar{K}}{R_{0}^{2}} .
$$

$\gamma$ is the sum of $\gamma_{\mathrm{c}}$ and of a dispersion entropy contribution for which many different expressions were proposed. We will use [17]

$$
\gamma_{\mathrm{c}}=\frac{k T}{4 \pi R_{0}^{2}} \ln \phi .
$$

The sum of $\gamma_{\mathrm{c}}$ and $\gamma_{\mathrm{e}}$ has been calculated for the $\mathrm{C}_{10} \mathrm{E}_{4}$ system assuming $\bar{K}=0$. The data are reported in table I. Although the $\gamma_{\mathrm{e}}$ value might be incorrect, it is not expected to depend on $K$ and $\bar{K}$. The comparison with the experimental values indicates that $\bar{K}$ might indeed be negative and that it increases in absolute value when approaching the three phase region. Further work is underway to clarify this problem.

Let us finally note that $K$ and $\bar{K}$ can also be deduced from neutron scattering spectra of the droplet microemulsions [23]. The corresponding experiments are currently underway in the above non-ionic systems.

\section{Conclusions}

There is experimental evidence that the interfacial tensions between microemulsions and oil or water excess phases are related to the characteristic size in the microemulsion phase through the simple relations $\gamma R^{2} \sim k T$ and $\gamma \xi^{2} \sim k T$. Theories predict that $\gamma$ should rather be related to the bending elastic constants of the interfacial layer. The agreement with the above simple relations is probably due to the fact that the elastic constants are of order $k T$ in microemulsion systems.

Experimental determinations of the bending elastic constant $K$ from ellipsometry [16] together with experimental determination of droplet radii from phase boundary [14] allow to calculate the interfacial tensions for droplet microemulsions. The agreement between the calculated and experimentally 
determined interfacial tensions is good. However, the product $\gamma R^{2}$ varies more rapidly with temperature than predicted by theories. But these theories have neglected the role of the Gaussian bending elastic modulus, and its influence needs to be investigated. Further studies of droplets microemulsions could be helpful in this matter.

The interest of the description of the surfactant film energy in terms of elastic bending energy is further supported by experimental determinations of bicontinuous microemulsion microstructures where it is found that the maximum size is close to the persistence length of the surfactant film.

\section{References}

[1] P.G. de Gennes and C. Taupin, J. Phys. Chem. 86 (1982) 2294.

[2] D. Langevin, Accounts Chem. Res. 21 (1988) 255.

[3] S.A. Safran and L.E. Turkevich, Phys. Rev. Lett. 50 (1983) 1930.

[4] W. Helfrich, Z. Naturforsch. 28a (1973) 693.

[5] J. Jouffroy, P. Levinson and P.G. de Gennes, J. Phys. (Paris) 43 (1982) 1241.

[6] B. Widom, J. Chem. Phys. 81 (1984) 1030.

[7] W. Helfrich, J. Phys. (Paris) 46 (1985) 1263.

[8] L. Peliti and S. Leibler, Phys. Rev. Lett. 54 (1985) 1690.

[9] S.A. Safran, D. Roux, M.E. Cates and D. Andelman, Phys. Rev. Lett. 57 (1986) 491.

[10] B. Widom, J. Chem. Phys. 84 (1986) 6943.

[11] K.A. Dawson, Phys. Rev. A 35 (1987) 1766.

[12] G. Gompper and M. Schick, Phys. Rev. Lett. 62 (1989) 1647.

[13] M. Kahlweit, R. Strey and P. Firman, J. Phys. Chem. 90 (1986) 671.

[14] M. Kahlweit, R. Strey and G. Busse, J. Phys. Chem., submitted.

[15] S.T. Milner, S.A. Safran, D. Andelman, M.E. Cates and D. Roux, J. Phys. (Paris) 49 (1988) 1065.

[16] L.T. Lee, D. Langevin, J. Meunier, K. Wong and B. Cabane, Prog. Coll. Pol. Sci., to appear.

[17] J. Meunier, J. Phys. (Paris) 48 (1987) 1819.

[18] B.P. Binks, J. Meunier, O. Abillon and D. Langevin, Langmuir 5 (1989) 415.

[19] I. Szleifer, D. Kramer, A. Ben-Shaul, D. Roux and W.M. Gelbart, Phys. Rev. Lett. 60 (1988) 1966.

[20] D. Guest, L. Auvray and D. Langevin, J. Phys. Lett. 46 (1985) 1055.

[21] O. Abillon, B.P. Binks, C. Otero, D. Langevin and R. Ober, J. Phys. Chem. 92 (1988) 4411.

[22] V. Luzzati, P. Mariani and T. Gulik, in: Physics of Amphiphilic Layers, J. Meunier, D. Langevin and N. Boccara, eds. (Springer, Berlin, 1987).

[23] S.A. Safran, private communication. 